\title{
EKT ePublishing: Developing an Open Access publishing service for the Greek research community
}

\author{
Alexandros Nafpliotis, Victoria Tsoukala, Nikos Houssos, Andreas Kalaitzis and Evi Sachini* \\ National Documentation Centre/National Hellenic Research Foundation, Athens, Greece
}

\begin{abstract}
The present contribution concerns a case study of open access scholarly publishing in Greece, its history and effect in helping the local researcher community transition from a print-only mode of work to online working environments and in rendering Greek publications and scholarship more relevant to the international scholarly community. The paper elaborates on the goals of the project and the challenges that were encountered and addressed during its implementation. The project, which started in 2007 with the transition of three print journals in the humanities to an online and print format and online working environment, culminated in the development of an online platform that provides access to content and services from a single point in the web, ePublishing.ekt.gr. As part of the National Documentation Centre (EKT)'s services, we systematize and upgrade the journals' policies according to international standards, provide an online working platform and training, digitize and release in open access academic articles (more than 3,500 articles in established journals, published by small, non-profit, academic/scholarly society publishers, so far), provide DOIs, as well as concentrate on electronic books and conference proceedings - also to include purely online books in the future, starting with a born-digital monograph in a Humanities subject (onlineBook). In a nutshell, we have focused on providing publishers of scientific journals a range of comprehensive services which are constantly updated and improved in the light of the developments in scholarly communication, and which foster the internationalization, visibility, and preservation of research in these fields.
\end{abstract}

Keywords: Electronic journals, Open Access, Greece, Open Journal Systems (OJS), ePublishing

\section{Introduction}

The present paper presents a case study of open access scholarly publishing in Greece, its history and effect in helping the local researcher community transition from a print-only mode of work to online working environments and in rendering Greek publications and scholarship more visible and more relevant to the international scholarly community. The paper elaborates on the goals of the project and the challenges that were encountered and addressed during its implementation. One of the main reported successes of the project is the increased awareness among Greek researchers of the capabilities and potentials of modern scholarly communication systems and the creation of a demand originating from the corresponding research communities themselves for the continuation and expansion of similar activities in the future. It should be noted here that most current content belongs to various fields of the Humanities and the Social Sciences; the significance of this cannot be overstated, especially for regional studies, often in the Greek language. In this respect, the contribution to this particular community in Greece is

\footnotetext{
${ }^{*}$ Corresponding author: Evi Sachini, National Documentation Centre/National Hellenic Research Foundation, Leoforos Vassileos Konstantinou 48, 11635, Athens, Greece. E-mail: esachin@ekt.gr.
} 
very important. Notwithstanding that, it should be stressed that our services and content are not limited to these specific scientific fields, but are also addressed to a wide variety of academic and scholarly publications from across the disciplines.

\section{History of the service}

The scholarly ePublishing service has been developed for and in collaboration with the Greek research community by the National Documentation Centre (EKT at www.ekt.gr), the backbone organization of the Greek national infrastructure for scientific documentation, online information and support services for research, science and technology. EKT is the national institution for documentation, information and support on science research and technology issues. Founded in 1980, EKT is part of the National Hellenic Research Foundation (NHRF). The latter also comprises three research institutes, one in Humanities (Institute for Historical Research) and two in the Natural Sciences (Biology/Biotechnology, Theoretical and Physical Chemistry). The platform was created as part of a wider project for the implementation of repositories and electronic publishing and was co-funded by the EU and the Greek state. More specifically, EKT's e-infrastructure development was co-financed by Greece and the European Union/European Regional Development Fund (Operational Program "Digital Convergence") project, called the National Information System for Research and Technology (NISRT). The central scope of the project was to increase support for OA in the Greek research and academic community by establishing infrastructures such as repositories and electronic journals that afford the digitization, permanent storage and free world-wide dissemination of the scientific output produced at NHRF (EKT also developed the IR of NHRF, Helios, Pandektis, A Thesaurus of Primary Sources for Greek History and Culture and the National Archive for PhD Theses).

The project, which started in 2007 with the transition of three print journals in the humanities to an online and print format and online working environment, culminated (in early 2013) in the development of an online platform that provides access to content and services from a single point on the web, http://epublishing.ekt.gr. It is important to stress that EKT's ePublishing platform constitutes unique service in Greece, providing open access scholarly content and professional services to the academia, publishers and the wider public. As part of EKT's services, we systematize and upgrade the journals' policies according to international standards, provide an online working platform and training, digitize and release in open access academic articles (more than 3,500 articles in 15 established journals, published by small, non-profit, academic/scholarly society publishers, so far), gradually provide DOIs, and concentrate (apart from articles) on books and conference proceedings - also to include purely online books in the future, starting with a born-digital monograph in a Humanities subject (onlineBook).

\section{EKT ePublishing: aims and services}

We believe that the primary purpose of scholarly communication is the promotion and distribution of knowledge and we are committed to the principles of open access in providing publishing services (software, tools, knowledge, technical expertise, consultation services, web hosting and documentation) to the country's scientific communities, organizations and institutions. More than a dynamic application, EKT ePublishing embodies a business process which reinforces EKT's vision to contribute to the process of transition to new models of e-science. Adhering to the principles of Open Access to scientific information, EKT ePublishing services enable the transition of prestigious scientific publications into 
an online mode of operation. At EKT, we work together with publishers, editors and authors to deliver the electronic edition of accredited journals, books and conference proceedings so that they are openly available to the research and academic community in Greece.

At all stages of the publication process, EKT ePublishing supports publishers, institutions and research bodies with a range of comprehensive services which are constantly updated and improved in the light of the developments in scholarly communication. Services include, most significantly, the organization, documentation and organized dissemination of metadata and content of scholarly journals, training and consulting services on issues such as the standardization of editorial processes according to internationally accepted standards, intellectual property, the inclusion of content and metadata in international content indexers and harvesters via interoperable systems, and retroactive digitization and ingestion of legacy digital content into the platform as well as production of metadata for past issues.

We also offer open source interoperable technology and continuous IT support to the publishers we are working with. ePublishing services are addressed to public institutions and publishers of accredited scholarly journals as well as to the wider public.

\section{Technical platform and services}

In terms of creating a dedicated e-publishing tool for our eJournals, Open Journal Systems (OJS, by far the most popular open source platform at the international level) was identified as the most suitable system. We decided on customizing the OJS platform in ways that meet the requirements of each journal implementation. Some of the modifications developed on OJS were of the following types: additions and enhancements to the article and user metadata, including full support for hierarchically controlled vocabularies; functionality and journal workflow customisations; online reading through a sophisticated image server infrastructure; batch importing of previous journal issues; layout, appearance and usability enhancements; handling the case of articles that are complemented with supplementary files (e.g., images); multilingual support.

EKT's ePublishing web platform itself was developed in late 2012 using a distributed architecture based on the Drupal framework, the Solr indexing engine, the Apache Tika content analysis platform and the multiple OJS e-journal installations. The majority of content of EKT the ePublishing platform is harvested and published from external e-journal instances, with authorized users also having the right to manually import content directly into the system. The connection between EKT ePublishing platform and the various OJS web platforms is accomplished through appropriate REST style web services.

The platform was customized in order to showcase the different categories of publications (eJournals, eBooks, eProceedings) in the most effective and presentable way. Our eJournals are presented in the form of a directory, with every journal being accompanied by basic information (focus and scope, scientific fields covered, editorial policies and author guidelines, announcements etc.) and, of course all the articles, grouped in issues and presented in PDF format within the platform itself. There are also separate directories for eBooks and eProceedings, with each publication both available for online reading through a page-by-page viewer (using a special infrastructure and set of services developed by EKT's Information Systems department [12]) and to download in PDF format. An important functionality of the ePublishing platform is the index of authors, which helps the reader to easily find all the works penned by the same author from across all electronic publications on the platform.

The EKT ePublishing platform allows electronic publications' PDF files to be uploaded in the corresponding installation file system. As for the electronic publications that are imported in the platform 
from external electronic journals systems, the source files of the publications are not transferred to the platform's file system, for better performance and statistics management. Instead, the full-text content is retrieved on demand for download or online viewing.

As a result, not only the local files that are manually uploaded to the platform, but also remote PDF files from various external web platforms, are indexed in the same search platform, enabling distributed full text search in EKT ePublishing with hit highlighting and faceted search, among other features.

An important feature of the EKT ePublishing platform is full-text search to all the material that is presented through the platform, including not only the books and proceedings that are hosted within the system but also the text of journal publications which reside in the individual e-journals (separate instances of OJS). Locally and remotely stored files are processed in the same central index, implemented using the Solr platform. A system of automatic extraction of textual content from PDF files and periodic synchronization with the central index ensures that the search function is updated when full-text material is inserted or modified within journals. The Apache Tika tool has been integrated in the Solr platform to achieve text extraction. Furthermore, hit highlighting in search results has been implemented utilizing and integrating the relevant facilities of Solr and Drupal.

\section{Content and impact}

Currently ePublishing hosts quality-assured scientific content from 13 Greek scientific publishers (the Institute of Historical Research of the National Hellenic Research Foundation, the National Centre for Marine Research, the Christian Archaeological Society and other respectable academic or scholarly publishers). The platform includes 13 peer-reviewed Open Access journals (following scientific evaluation processes and indexed in international databases) - each with its own, dedicated OJS installation - as well as 2 other scientific publications, comprising more than 3,500 scientific articles in various languages, and more than 50 e-books and conference proceedings. Readers can browse an index of about 3,000 authors or search for material that interests them by choosing from an extensive list of articles and books in 54 scientific fields. The integrated ePublishing environment has been developed with open source interoperable technology and, therefore, feeds other platforms and portals, such as www.openarchives.gr, the largest portal providing a single point of access to Greek scientific and cultural digital content of high quality (also developed by EKT). The platform is constantly updated with new issues, journals and other publications, as well as new services, and EKT is expanding its collaborations with scientific publishers who are active not only in the Humanities and Social Sciences but in other scientific fields, as well. As far as the promotion of the publications is concerned, we have developed a dissemination strategy for wide variety of outlets and recipients, for example creating leaflets and emailing them to lists and individuals with specialized interests; networking; presenting in conferences; and using social networking capabilities (on blogs, Facebook, Twitter, Flipboard etc.).

The statistics on the use of the journals are encouraging. They show a steady interest in them, for the moment centered in, but not exclusively focused on Greece. Greek and foreign researchers use the journals in their new form to conduct research. A noteworthy fact is that a more or less important part of the traffic for all journals originates from outside Greece, ranging from one-third to two-thirds of the visits. A large number of countries are represented, demonstrating the power of electronic publication in the worldwide dissemination of content. More focus will be directed in the promotion of the journals abroad, aiming at an increase of their significance as research tools for the global scholarly community. Our efforts towards achieving that goal (including targeting specific scholarly groups and communities) 
have already borne some fruit, with statistics for January 2014 showing a large increase in access from countries other than Greece, in comparison to figures for the first year of the platform's operation (approx. $33 \%$ vs $13 \%$ ). As far as the impact of specific journal pages is concerned, there has been a steady growth in registered users for our eJournals, as well as in numbers for visits per year for all ePublishing journals, with 3,700 unique users per month on average for each eJournal.

\section{New services and future plans}

Since 2008, when EKT's ePublishing begun with the launch of one journal, this activity has now developed into a full-blown service that continuously grows. With a view to improving our services, increasing and diversifying the publishers served through ePublishing and the content that becomes available, new services are planned and the future of EKT ePublishing is seen in the context of relevant European services and infrastructures. Imminently, a dedicated HelpDesk will be developed and available in the summer of 2014. There, registered users will be able to submit electronically their requests and track their status, receive the response and even access the history of the communication with EKT. All users visiting the HelpDesk will be able to find answers to the most common questions that arise from new and old EKT ePublishing users by visiting the "FAQs". Users will be able to leave a comment or some input, which does not require a reply or support from EKT's personnel, by filling-in the specifically created form.

Further, EKT is seeking to enrich the types of publications offered through ePublishing. A major endeavor that will begin in the fall of 2014 is the onlineBook service, a specialized electronic publishing service for open access monographs. While this, as all ePublishing services, is offered to the entire research and publishing community of the country, the onlineBook is being planned specifically with the needs and scholarly communication trends of the Social Sciences and Humanities research communities in mind [7]. This service is expected to be of particular interest for these scientific fields, since the monograph is a significant means for communicating their in-depth research. The aim of the onlineBook service is to enable publishers and Greek scientists to publish digital-born cutting-edge peer-reviewed research monographs in open access (also providing the ability to print on demand). It will comprise a suite of services for the publishers, namely, consulting that will help them develop systematic policies and specifications and improve their processes, as well as technical for the publication and dissemination of their work. As a first step, pilot-publications will be initiated with publishers already collaborating with EKT ePublishing. In 2015, it is expected that the service will become more widely available to accredited publishers. We are, additionally, exploring the possibilities of experimenting with the implementation of an open access data journal, in order to provide help the research and academic community transition to a culture of sharing their research data.

Conclusively, EKT ePublishing has gradually and steadily grown since its inception in 2007 to become a unique service for the Greek research and education community. The recent launch of the single access point platform, http://epublishing.ekt.gr, in the beginning of 2013, further contributed to the wide dissemination of the service among researchers and publishers in Greece. Apart from specific plans that aim at expanding collaborations with Greek publishers, diversifying types of publications and extending the technology, EKT is aware of the need to be part of international networks and of e-infrastructures for conducting and communicating research and is thus swiftly moving towards this direction. Finally, particular emphasis is placed on exploring appropriate income models that will enable a sustainable growth of the service in the future as one directed to accredited Greek not-for profit scientific publishers. 
This should be based on diversifying its incoming resources and increasing inclusion of the full spectrum of the Greek academic and research community, which it has been developed to serve (universities, research centers, and scientific societies).

\section{Note}

On this, see the recommendations of 'Open Access Monographs in the Humanities and Social Sciences Conference Report' by Jisc Collections and OAPEN, and specifically the one stating that '[W]e need incentives to encourage researchers to embrace OA enough to digitize and make available their primary sources and to embrace the opportunities that technology provides to make the future of the book a truly interactive and collaborative venture', available online at: jisc-collections.ac.uk/reports/oabooksreport.

\section{References}

[1] Apache Solr, http://lucene.apache.org/solr/.

[2] Apache Tika, http://tika.apache.org/.

[3] Drupal, https://drupal.org/about.

[4] J. Houghton, B. Rasmussen, P. Sheehan, C. Oppenheim, A. Morris et al., Economic Implications of Alternative Scholarly Publishing Models: Exploring the Costs and Benefits, 2009, 256 pp., available at: http://ie-repository.jisc.ac.uk/278/ [Accessed January 26, 2014].

[5] Institutional repository of NHRF, Helios, available at: http://helios-eie.ekt.gr/.

[6] M. Laakso, P. Welling, H. Bukvova, L. Nyman, B.-C. Björk et al., The development of open access, journal publishing from 1993 to 2009, PLoS ONE 6(6) (2011), e20961.

[7] National Hellenic Research Foundation (NHRF), www.eie.gr.

[8] National Information System for Research and Technology (NISRT), www.epset.gr.

[9] Open Journal Systems (OJS), http://pkp.sfu.ca/ojs/.

[10] Pandektis, A thesaurus of primary sources for Greek history and culture, available at: http://pandektis.ekt.gr/.

[11] E. Sachini, V. Tsoukala, N. Houssos, I.-O. Stathopoulou, Ch.-E. Paschou and A. Paraskevopoulou, Open Access in the humanities: a case study of developing three open-access electronic journals, Greece, in: 13th International Conference on Electronic Publishing: Innovation in Communication Paradigms and Technologies, Milan, Italy, 10-12 June 2009, pp. 543-556.

[12] P. Stathopoulos, N. Houssos, O. Stathopoulou, G. Stavrou, V. Soumplis and A. Enhancing, OJS journals with advanced online reading and viewing capabilities, in: PKP Scholarly Publishing Conference, 2011.

[13] The National Archive for Ph.D. Theses, http://phdtheses.ekt.gr.

[14] V. Tsoukala and V. Sachini, E-journal and open access journal publishing in the humanities: Preliminary results from a survey among Byzantine studies scholars', in: Proceedings International Conference on Integrated Information, Vol. 29, Kos, September 3, 2011. 